\section{Edycaçäa

Maria Inês Côrte Vitoria PUCRS, RS, Brasil

\section{Equipe Editorial}

Pricila Kohls dos Santos PUCRS, RS, Brasil

Marcelo Oliveira da Silva PUCRS, RS, Brasil

Carla Spagnolo

PUCRS, RS, Brasil

Rosa Maria Rigo

PUCRS, RS, Brasil

\section{ISSN 2179-8435}

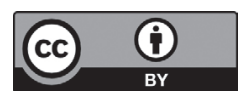

Este artigo está licenciado sob forma de uma licença Creative Commons Atribuição 4.0 Internacional, que permite uso irrestrito, distribuiçaao e reproduçāo seja corretamente citada. http://creativecommons.org/licenses/by/4.o/deed.pt_BB

\title{
Experiencia de internacionalización y desarrollo humano entre Colombia y Brasil
}

\author{
Experiência de internacionalização e desenvolvimento humano \\ Experiência de internacionalização e desenvolvimento humano
entre Colômbia e Brasil \\ Internationalization experience and human development between Colombia-Brazil
}

Adriana Pineda Robayo ${ }^{a}$

RESUMEN: La internacionalización, como parte de los procesos culturales, utilizados por las Instituciones de Educación Superior en el mundo, para establecer redes de intercambio, articulación y cooperación, se integra paulatinamente a sus funciones sustantivas de docencia, investigación y extensión, y está orientado a generar mayores niveles de competitividad global. Teniendo en cuenta estos elementos, y utilizando el modelo de investigación basado en la noción de experiencia vivida, propuesto por Van Manen (2003), se sustenta en el presente análisis, la importancia de hacer visibles las implicaciones ontológicas que entraña, este proceso, ya que producto del mismo, es posible evidenciar aspectos fundamentales en el desarrollo humano, como la re significación de la realidad, los saberes, la personalidad y la formación profesional. El artículo es producto de la experiencia vivida en procesos de internacionalización propiciados en diferentes ámbitos de la formación profesional (pasantía doctoral, posdoctorado), entre instituciones de Brasil y Colombia, y demuestra la importancia de asumir la internacionalización, como una instancia necesaria para propiciar el desarrollo humano y como un proceso de continua construcción del ser, a través de la dinámica que supone el conjunto de relaciones que propicia, con sigo mismo, con los demás y con la cultura.

Palabras clave: internacionalización; desarrollo humano; experiencia vivida.

RESUMO: A internacionalização, como parte dos processos culturais, utilizados pelas Instituições de Educação Superior no mundo, para estabelecer redes de intercâmbio, articulação e cooperação, se integra paulatinamente a suas funções substantivas de docência, investigação e extensão, e está orientado a gerar maiores níveis de competitividade global. Levando em consideração estes elementos, e utilizando o modelo de investigação baseado na noção de experiência vivida, proposta por Van Manen (2003), se sustenta na presente análise, a importância de tornar viáveis as implicações ontológicas que entranha, este processo, uma vez que produto do

\footnotetext{
* Doctora en Educación por la Red de Universidades Públicas de Colombia. Estudios Post-doctorales en Centro Universitario La Salle - UNILASALLE con beca PNPD/CAPES-Brasil. <adripineda10@hotmail.com>.
} 
mesmo, é possível evidenciar aspectos fundamentais no desenvolvimento humano, como a ressignificação da realidade, os saberes, a personalidade e a formação profissional. Este artigo é produto da experiência vivida em processos de internacionalização propiciados em diferentes âmbitos da formação professional (estágio doutoral, pós-doutorado), entre instituições do Brasil e Colômbia, e demostra a importância de assumir a internacionalização, como uma oportunidade para propiciar o desenvolvimento humano e como um processo de contínua construção do ser, através da dinâmica que supõe o conjunto de relações que propicia, com sigo mesmo, com os demais e com a cultura.

Palabras-chave: internacionalizacão; desenvolvimento humano; experiência vivida.

ABSTRACT: Internationalization, as part of cultural processes, used by higher education institutions in the world, to stablish new exchange networks, coordination and cooperation is gradually integrated into its substantive functions of teaching, research and extension, and is aimed at generating higher levels of global competitiveness. Taking into account these elements, and using the research model based on the notion of lived experience, proposed by Van Manen (2003), is exposed on this analysis, the importance of making the ontological implications involved visible, this process, that product itself, it is possible to highlight fundamental aspects of human development, such as re meaning of reality, knowledge, personality and professional education. The article is the result of the experience in internationalization processes brought about in different areas of vocational training (doctoral internship, Ph.D.), between institutions in Brazil and Colombia, and demonstrates the importance of taking internationalization as a necessary instance to promote the human development and a process of continuous construction of being, through the dynamics of the relationships that allows, with himself, with others and with culture.

Keywords: internationalization; human development; lived experience.

\section{Introducción}

T a internacionalización para las Instituciones de Educación Superior (IES), es en esencia, una herramienta necesaria en la posibilidad de participar activamente del dialogo con una sociedad globalizada, ya que como lo admite la normatividad del estado colombiano (MEN 2009), "este proceso le otorga una dimensión internacional e intercultural a los mecanismos de enseñanza e investigación de la educación superior, a través de la movilidad académica de estudiantes, docentes e investigadores".

Los procesos de internacionalización en la formación profesional, se configuran como espacios de realidades claramente relacionales, donde la dinámica establecida directamente entre los seres que de ella participan a través de la comunicación, plantea el reencontrarse y reconstruirse continuamente de un yo que es por y para los demás. 
Procesos como la suscripción de convenios interinstitucionales, la estructuración de redes académicas, la internacionalización del currículo, la utilización diversificada de tecnologías de la información y la comunicación, la estructuración de políticas de cooperación y acuerdos interuniversitarios con el apoyo de instancias como el MERCOSUR, son algunos de los mecanismos, desde los cuales las IES, a la par que "amplían sus espacios de acción, propician el mejoramiento en los estándares de acreditación y evaluación de la calidad de los programas académicos y las instituciones en Colombia" (MEN 2009).

Igualmente en el contexto del Brasil, la internacionalización en la educación superior es concebida como eje de integración y articulación de las dimensiones pedagógica y estratégica de gestión en las universidades. En este sentido Fossatti (2015) señala que a partir de diversas investigaciones, es posible verificar como la internacionalización actualmente hace parte de los Planes de Desarrollo Institucional, como una estrategia importante para en el avance de las Instituciones brasileras de Educación Superior.

Lo anterior sustentado en el objetivo de responder adecuadamente a los requerimientos de los estándares de calidad, y a la demanda de IES, que como lo plantea De Sousa (1994, p. 239), "puedan trascender de la rigidez institucional y la dificultad para captar las señales del mercado de trabajo, hacia propuestas de formación centradas en la flexibilidad, el desarrollo personal y la motivación individual".

Frente a este reto, universidades de Brasil y Colombia han estructurado procesos de internacionalización, que de acuerdo con Landinelli (2010), transitan desde la valoración de la producción local, hacia la construcción de procesos globales e interculturales. Estos facilitan el establecimiento de relaciones y la vivencia de experiencias a través de las cuales se construyen saberes como producto de un proceso interactivo donde el lenguaje y la educación con sus herramientas simbólicas, abstractas y verbales le dan forma a nuevos conocimientos.

El presente artículo, es producto de la experiencia vivida en el proceso de internacionalización, propiciados en diferentes ámbitos de formación (pasantía doctoral, posdoctorado), entre instituciones de Brasil y Colombia, y demuestra la importancia de asumirla, como una instancia necesaria para propiciar el desarrollo humano y como un proceso de continua construcción del ser, a través de la dinámica que supone el conjunto de relaciones que establece, con sigo mismo, con los demás y con la cultura.

\section{Desarrollo humano}

Los planteamientos de los documentos publicados desde 1999 hasta el 2015 por el Programa de las Naciones Unidas para el Desarrollo, muestran un discurso relacionado con el desarrollo humano consecuente con las ideas de Sen (2000), quien plantea que este, trasciende los límites del campo económico y está orientado a lograr que los 
hombres y mujeres de cada país, gocen de las condiciones necesarias para desarrollar su potencialidades y vivir creativa y productivamente, brindándoles una gama de oportunidades y opciones diversas para ser, saber y hacer.

Igualmente el concepto de libertad como eje central y como atributo clave en el repertorio de capacidades que debe desarrollar el ser humano para alcanzar una vida digna, coinciden con las ideas de Nussbaum y Rodil (2010), quienes encuentran, un punto de convergencia de los mismos en la posibilidad real de establecer procesos de educación centrados en la interacción efectiva mediada por la comunicación.

Estas instancias le permiten al hombre expresar, pensar, comprender, hacer y sentir, en un contexto de globalización en el que la extensión del capitalismo, exige la estructuración de nuevas propuestas que apunten a la formación de seres humanos respetuosos, éticos y sobre todo que conozcan y participen con sus vivencias, de los valores necesarios para vivir en democracia.

A la par, pensadores como Max-Neef, Elizalde y Hopenhayn, (1998, p. 20), proponen un desarrollo a escala humana, entendido como el establecimiento de relaciones armónicas entre diferentes instancias del ser humano, la satisfacción de sus necesidades básicas, el fortalecimiento de su capacidad de auto dependencia y la generación de procesos desde los cuales los sujetos logren la articulación coherente y sustentable con su entorno natural, con la tecnología de los procesos globales y con la dinámica de los comportamientos locales, "de lo personal con lo social, de la planificación con la autonomía y de la sociedad civil con el estado".

En este sentido Bronfenbrenner (1999), reitera la importancia del establecer relaciones efectivas, regulares y reciprocas, por periodos de tiempo prolongados, como factores esenciales para el desarrollo humano, así como la participación en actividades que se complejizan gradualmente. En ellas el sujeto tiene la posibilidad de vivir experiencias académicas sociales y culturales que garantizan un abordaje bioecológico del desarrollo humano, a partir de la articulación armónica de cuatro dimensiones: el proceso, la persona, el contexto y el tiempo, que en su interacción garantizan mayores niveles de comprensión de la realidad.

Es decir, se ratifica el planteamiento de la construcción de humanidad a través del establecimiento continuo de relaciones mediadas por la comunicación con los demás, con el contexto cultural y con nosotros mismos, como medio para adoptar una propia posición frente al mundo, en la estructuración del yo en relación con el otro, en el que se encuentra, se conoce, se percibe y existe, ya que como lo plantea Hoyos $(2002$, p. 30) "la educación es comunicación y la comunicación constituye ciudadanía y es, por tanto, punto de partida para la comprensión y la práctica de la democracia".

Los elementos enunciados a partir del concepto de desarrollo humano, coinciden con la perspectiva del análisis desde la cual, la vivencia efectiva del proceso de internacionalización, más allá de constituirse en una experiencia de orden académico, tiene importantes implicaciones ontológicas, desde las cuales según Heidegger (1997), el hombre 
de manera natural se interpreta, construye otras comprensiones de humanidad, y nuevas perspectivas frente al estar en el mundo.

Este mundo de la vida, desde el que se estructura el proceso de internacionalización ofrece la posibilidad de pensar, conocer y actuar comunicativamente a partir de las interacciones que se establecen. A su vez facilita la comprensión de diversas situaciones, la aceptación del otro como realidad comunicativa en relación con el yo, en la que el descubrimiento de hacer parte de una comunidad, posibilita la construcción de propias representaciones del mundo.

La re-significación del ser como único, con una especificidad personal activa, biológica, psíquica, social y cultural en expansión, se hace visible en el contexto de la internacionalización, al sabernos miembros de una comunidad con diversas creencias, valores, interpretaciones y significados.

Esta conexión entre multidimensionalidad del ser, diversidad y complejidad de la realidad, nos enfrenta al planteamiento de Prigogine (1996) desde el cual, el hombre se transforma, evoluciona en la dinámica de las fluctuaciones presentes en la coexistencia entre naturaleza del ser y realidad, donde nacen nuevas soluciones, nuevos equilibrios nuevas estructuras, que reflejan y la vez son producto de la interacción entre el mundo exterior, y del papel constructivo del tiempo.

\section{Globalización e internacionalización}

El crecimiento y la transformación que el planeta ha experimentado en los últimos años, a causa de los procesos de globalización, ha impactado en todos los ámbitos de la vida del ser humano, pero en especial en la educación. Los avances en la ciencia y la tecnología han propiciado según Hudzic (2008), transformaciones importantes en la economía, la política y las relaciones interpersonales, y han eliminado los obstáculos que persistían para el intercambio de ideas, conocimientos y relaciones sociales.

Estas transformaciones, a su vez dan paso a una nueva forma de ver el mundo y la realidad, en la que diversidad y complejidad se conectan con las condiciones de sufrimiento y desgaste infringidos a la naturaleza y se valora cada vez más la habilidad del ser humano para asumirse como parte de un todo, al que debe integrarse desde su propia diferencia.

Las modificaciones implícitas en estos procesos, llevan al ser humano a reflexionar sobre su existencia y su papel en el mundo globalizado, en el que además de conocimientos técnicos y específicos de cada área, y el dominio de otros idiomas, persiste la necesidad de formar un ser con las competencias adecuadas para integrarse a esa nueva forma de sociedad y de cultura. En este sentido Stallivieri (2009), menciona que el estudiante global necesita estar 
preparado para entender al otro a través de las semejanzas y diferencias al mismo tiempo en que se reconoce a través de este contacto, aprende y se enriquece como ser humano.

Es preciso reafirmar el papel de las universidades, en la formación del ciudadano global, ante el cual es preciso observar dos perspectivas que según Knight (2012), permiten verificar la labor de las IES, en torno a la Internacionalización. Una, desde el interior de la institución y que centra su interés en la internacionalización del currículo, el desarrollo de procesos investigativos en colaboración con universidades de otros países y la recepción de estudiantes a través de convenios de intercambio.

Esta perspectiva es coherente con los planteamientos de Mota (2009), quien afirma la alta valoración que el contexto laboral globalizado, otorga a los profesionales que han desarrollado estudios en el exterior, ya que se consideran más flexibles y cualificados en las competencias interculturales, aspectos que hacen que la movilidad académica sea considerada fundamental en el proceso de formación.

Otra arista del proceso de internacionalización, es la que se da a través de la educación transfronteriza, que de acuerdo con Knight (2006. p. 19), busca la proyección de su función de docencia hacia el exterior a través de procesos de movilidad académica de "profesores, estudiantes, programas, proveedores, conocimientos, ideas, proyectos y servicios". El autor plantea, que este proceso ha posibilitado la consolidación de nuevos referentes, en torno a la Educación superior, donde las universidades virtuales con sus sedes en diferentes países, a la vez que responden a las necesidades de formación, también aprovechan los avances tecnológicos.

Esta modalidad de Internacionalización ha sido ampliamente analizada por organismos internacionales como la Organización para la Cooperación y el Desarrollo Económico OCDE (2005) y la UNESCO quienes plantean las directrices en torno a la calidad de los procesos educativos transfronterizos, y a su posibilidad de moverse entre la presencialidad y la virtualidad, a través de herramientas tecnológicas.

\section{La experiencia vivida}

A partir de las elaboraciones conceptuales presentadas anteriormente, la propia experiencia frente al proceso de internacionalización, aflora como parte de una construcción personal, que al ser compartida, aspira a convertirse en experiencia para otras personas.

Hablar de la experiencia vivida en el proceso de internacionalización, como "acontecimiento de vida", más allá de ofrecer explicaciones causales, intenta construir un relato sobre la vivencia del mismo.

La experiencia vivida, como elemento fundamental en la ciencia fenomenológica, desde la que se ratifica su carácter temporal, reflexivo y significativo, en tanto que implica la totalidad de la vida, es analizado por Van 
Manen (2003 p. 56), cuando menciona que "el examen interpretativo de la experiencia vivida, se caracteriza desde el punto de vista metodológico, por relacionar lo particular con lo universal, la parte con el todo, lo episódico con la totalidad".

¿Cómo me hice parte de este proceso? En frecuentes discusiones con colegas, el tema de estudiar en otro país, con sus maravillas y ventajas, se hacía presente como algo que definitivamente, no se encontraba en los planes inmediatos de desarrollo profesional. Argumentos económicos, laborales y familiares siempre estaban prestos a disolver cualquier inquietud frente al tema. En el año 2014, durante el desarrollo de una de las disciplinas de formación doctoral, conocí a una profesora de Brasil, quien había sido invitada para dictar un seminario.

La experiencia de conocerla y encontrar en su discurso y conocimiento muchos de los elementos que necesitaba en la pesquisa, fueron generando lentamente los argumentos necesarios para encaminar el proceso investigativo, hacia la posibilidad de profundizar en sus construcciones conceptuales. Fue así como al solicitar su participación como tutora internacional de mi tesis doctoral, se activaron desde su universidad en Brasil y desde la mía en Colombia, los mecanismos necesarios para estructurar un convenio interinstitucional, en el marco del cual me fue posible desarrollar un primer proceso de pasantía en Brasil, con una duración de un mes y cuyo objetivo era la organización del proceso metodológico de la investigación.

Esta descripción sobre el primer paso de la experiencia vivida, en el proceso de internacionalización, evidencia la importancia trascendental de la comunicación como instancia de relación, desde la cual un sujeto que se percibe y existe, encuentra nuevas posibilidades de humanización en la interacción con otra cultura y otras formas de pensar.

El contraste entre la idea inicial, frente a la dificultad del proceso de internacionalización, la lejanía de un proceso de formación en otro país y la experiencia de salir y conocer por la propia vivencia, son la base desde la cual es posible ratificar la existencia de unas implicaciones ontológicas. En este sentido Van Manen (2003 p. 60) afirma que "las preocupaciones fenomenológicas, unidas al carácter ontológico de la experiencia vivida, indagan por la naturaleza esencial del fenómeno experimentado en su significado pleno", y que progresivamente lleva a la re significación de la realidad del ser.

A partir de esta experiencia y de las construcciones logradas, en el primer proceso, la relación académica, dio paso a la consolidación de proyectos de producción bibliográfica conjunta, que a su vez, propiciaron la participación en eventos propuestos por la Asociación de Estudios Latino Americanos en Puerto Rico y Nueva York, así como la inclusión de ponencias en foros y seminarios realizados en Vancouver y Colombia.

Expresar el significado profundo de una experiencia vivida, a través de la reflexión sobre la misma, y su ubicación en el contexto del conjunto de experiencias humanas, se convierte desde los planteamientos de Van Manen 
(2003), en el camino propicio para explicar por extensión, las implicaciones que para el desarrollo humano tiene, participar en un proceso de internacionalización.

En el año 2015, ya concluido el doctorado y con grandes expectativas frente al trabajo conjunto con la IES de Brasil, se publica una convocatoria desde la cual el gobierno brasilero, a través del Programa Nacional de Post Doctorado otorga becas (PNPD), con el objetivo de promover la articulación de investigadores brasileros o extranjeros, en proyectos de investigación desarrollados por los programas de post-grado en el país.

Envié la documentación necesaria para participar del proceso, más por curiosidad que por convicción. La sorpresa fue muy grande cuando al consultar el resultado aparecía mi nombre como ganadora de la beca. Un sinnúmero de ideas, preocupaciones y preguntas llegaron a mi cabeza y se combinaban con la alegría y la satisfacción del haber recibido tal distinción, pero también con la angustia de enfrentar un reto, que llevaba consigo la necesidad de asumir grandes cambios en mi vida familiar y profesional, ya que se trataba de estar un año, viviendo lejos de casa.

Un aspecto trascendental en este proceso fue el apoyo de mi esposo e hijos quienes me animaron a asumir la experiencia. Sin duda era una prueba que la vida nos colocaba y ante la cual la tenacidad, fortaleza, valentía y solidaridad de cada miembro de la familia eran definitivas. La elección era difícil, pero era necesario afrontarla de tal manera que más allá de ser fuente de agonía, lograra ser efectivamente una oportunidad para celebrar lo especiales que somos como familia y las razones que rigen nuestras relaciones de padres, hijos y esposos.

El reto no era fácil, requería compromiso y habilidades de comunicación de cada miembro de la familia. El carácter temporal de la experiencia, asociado a la motivación de mejorar nuestras perspectivas de futuro se consolidaron como ejes del proceso. Igualmente la oportunidad para demostrar la capacidad de cada uno para estar solos, para ser valientes y para resolver las dificultades propias de la cotidianidad.

En esencia, la primera realidad que debió ser reacomodada fue la familiar, lo que se logró con la voluntad de encontrar nuevos espacios para compartir, comunicarnos y visitarnos.

La llegada a Brasil, fue sencilla, la universidad a través de los funcionarios de internacionalización, estuvo atenta en todo momento a acompañar los procesos administrativos propios de la reglamentación del país, y todo lo concerniente a la logística de acomodación, a través del despliegue de recursos humanos y técnicos necesarios en la resolución de situaciones relacionadas con alojamiento, transportes, servicios y procesos de acogida.

Algo que en principio resultaba intimidante era el tema del idioma, por cuanto no manejaba el portugués, al llegar encontré que, tanto la tutora como otros funcionarios de la universidad hablaban español, lo que facilitó mucho este primer proceso, y a la vez sirvió de soporte para asumir la responsabilidad de familiarizarme y aprender el idioma. 
Durante el primer semestre del año 2016, realice diferentes labores académicas que hacen parte de la investigación post doctoral, en los que fue posible aprender pero también aportar desde mi conocimiento y experiencia, de tal manera que el intercambio y la re significación de saberes fue elemento esencial de la experiencia.

Actividades e interacciones con niños de diferentes edades en una escuela pública, con profesores, estudiantes y egresados de programas de educación, con colegas y directivos de la universidad, ampliaron mi horizonte de relaciones a la vez que facilitaron el establecimiento o reafirmación de referentes sociales, culturales, políticos y pedagógicos.

Esta interacción armónica, en la que yo como sujeto activo y productivo, me articulaba con un contexto cambiante y rico en estímulos, en un proceso amigable pero exigente y por un tiempo prolongado, me permitieron corroborar los planteamientos de Bronfenbrenner (1999), en torno a la posibilidad de verificar procesos de desarrollo pleno del ser, que desde el presente análisis considero, de vital importancia compartir.

La visión integral, sistémica y naturalista del autor coincide con las comprensiones desarrolladas a través de la reflexión sobre la experiencia vivida, ya que se trata efectivamente de un proceso que en su complejidad, involucra diversos elementos ligados al contexto en el que se escenifica este desarrollo.

Esta visión ecológica del desarrollo humano, exige una constante de asimilación y acomodación de las relaciones e interacciones propiciadas entre los entornos que hacen parte del proceso. En este sentido el conocimiento de nuevos espacios sociales, académicos, culturales, políticos y el establecimiento de relaciones e interacciones con los mismos, fortalecen en el sujeto su habilidad para adaptarse a nuevas realidades. También permite verificar, la capacidad del nuevo contexto para adaptarse a las condiciones y necesidades, de quien llega como visitante, en el proceso de internacionalización, en una relación de reciprocidad constante.

Es evidente que la decisión de articularse a un proceso e internacionalización como el que se desarrolló entre universidades de Colombia y Brasil, implica para quien vive la experiencia, desplegar sus habilidades para adaptarse mental y emocionalmente, a una serie de cambios vertiginosos en lo que Bronfenbrenner (1979, p. 44), denomina su microsistema, su cotidianidad. Igualmente involucra la consolidación de nuevos mesositemas, o conjuntos de nuevos sistemas producto de la relación de intercambio y reciprocidad, propia de la incorporación a un nuevo entorno.

En este mismo sentido la experiencia permitió verificar la previsión por parte de la universidad de una serie de condiciones y recursos humanos y técnicos, que desde la universidad se desplegaron, como apoyo para quienes participan en el proceso de internacionalización, lo que repercute positivamente en la acogida al estudiante extranjero y coincide con lo que Bronfenbrenner (1979), denomino exosistema, movilizado y conectado con las condiciones necesarias para el desarrollo pleno del ser. 
Otro elemento relevante en la comparación entre el modelo sistémico de Bronfenbrenner (1979), y la posibilidad de generar desarrollo pleno e integral del ser, a través de su participación en procesos de internacionalización, propuestos por las universidades de diversos países, es la necesidad de fortalecer el macrosistema. Este, representado en políticas educativas, capaces de priorizar de los mecanismos necesarios, para incentivar la cualificación y la investigación, a través de la masiva participación en procesos formativos de alta calidad, ofrecidos por las universidades a escala global.

Desde este análisis es posible concluir que la observación cuidadosa de la relación entre los elementos propuestos, a partir del Modelo Ecológico, y las variables propias de cada sistema, serán elementos trascendentes para el desarrollo humano.

\section{Referencias}

BRONFENBRENNER, Urie. The ecology of Human Development. Cambridge: Harvard University Press, [19??]. (Trad. Cast.: La ecología del desarrollo humano. Barcelona: Paidós, 1979.)

BRONFENBRENNER, Urie. Environments in developmental perspective: theoretical and operational models. In FRIEDMAN, S. L. (Ed.). Measuring environment across the life span: emerging methods and concepts. Washington, DC.: American Psychological Association, 1999. p. 3-38.

FOSSATTI, Paulo; MIRANDA, José Alberto Antunes de; MOEHLECKE, Carolina. Internacionalização das IES brasileiras: uma análise crítica ante as dificuldades na gestão. INPEAU/UFSC, 2015. Disponble en: <https://repositorio.ufsc.br/handle/123456789/136050>. Acesso em: 11 mar. 2016.

DE SOUSA SANTOS, Boaventura. De la idea de universidad a la universidad de ideas. [S.1.]: [S.n.], 1994. Disponble en: <www. boaventuradesousasantos.pt/media/universidad>. Acceso en: 11 mar. 2016.

HEIDEGGER, Martin. Ser y Tiempo. Trad de Rivera Cruchaga, Jorge. E. Santiago: Ed. Universitaria, 1997.

HOYOS, Guillermo. Introducción a Edmund Husserl: renovación del hombre y de la cultura. Cinco ensayos. Barcelona/México: Anthropos/Universidad Autónoma Metropolitana, 2002. p. VII-XXXIII.

HUDZIK, John. Comprehensive internationalization. [S.1.]: Taylor \& Francis, 2008.

KNIGHT, Jane. Students Mobility and Internationalization: trends and tribulations. Research in Comparative and International Education, v. 7, n. 1, p. 20-33, 2012. <http://rci.sagepub.com/content/7/1/20.full.pdf+html>. <http://red.hypotheses.org/604>.

LANDINELLI, Jorge. Tendencias de la Educación Superior en América Latina y el Caribe. [S.1.]: [S.n.], 2010. Disponble en: $<$ http://www.iesalc.unesco.org.ve/index.php>. Acceso en: 11 mar. 2016.

MAX-NEEF, Manfred; ELIZALDE, A.; HOPENHAYN, M. Desarrollo a escala humana: conceptos, aplicaciones y algunas reflexiones. Barcelona: Icaria, 1998. 
MINISTERIO DE EDUCACION NACIONAL DE COLOMBIA. Internacionalización de la Educación Superior. 2009. Disponible en: <http://www.mineducacion.gov.co/1621/article-196472.html>. Acceso en: 11 mar. 2016.

MOTA, Keila. Turismo de Intercâmbio. In: Segmentação do mercado turístico: estudos, produtos e perspectivas. Barueri, SP: Manole, 2009.

NUSSBAUM, M. C.; RODIL, M. V. Sin fines de lucro: Por qué la democracia necesita de las humanidades. Madrid: Katz. 2010.

OCDE. Repaso a la enseñanza. Indicadores de la OCDE 2005. Disponible en: <https://www.oecd.org/education/skills-beyondschool/35317197.pdf>. Acceso en: 11 mar. 2016.

PRIGOGINE Ilya. El fin de las certidumbres. Barcelona: Editorial Andrés Bello, 1996.

SEN, Amartya;, RABASCO, E.; TOHARIA, L. Desarrollo y libertad. Barcelona: Planeta, 2000.

STALLIVIERI, Luciane. As dinâmicas de uma nova linguagem intercultural na mobilidade acadêmica internacional. Argentina, Universidad del Salvador - USAL, 2009. Disponível em: <http://www.researchgate.net/publication/228380071>. Acesso: 11 mar. 2016. VAN MANEN, Max. Investigación educativa y experiencia vivida. Barcelona: Idea Educación, 2003.

Recebido em: julho/2016

Aceito em: novembro/2016

\section{Endereço para correspondência:}

Adriana Pineda Robayo

Rua Santa Helena, 40

Canoas, RS, Brasil

<adriana.robayo@unilasalle.edu.br> 